\title{
IN SILICO STUDY OF CRYO-EM STRUCTURES OF ANTIGEN-ANTIBODY COMPLEX OF CHIKUNGUNYA FOR THE DEVELOPMENT OF DIAGNOSTIC AGENT
}

\author{
RINA FAJRI NUWARDA ${ }^{1,3}$, UMI BAROROH ${ }^{2,3}$, ZUHROTUN NAFISAH $^{2}$, BEVI LIDYA ${ }^{4}$, MUHAMMAD YUSUF ${ }^{2,3}$, \\ TOTO SUBROTO ${ }^{2,3 *}$
}

\begin{abstract}
${ }^{1}$ Department of Pharmaceutical Analysis and Medicinal Chemistry, Faculty of Pharmacy, Universitas Padjadjaran, Indonesia. ${ }^{2}$ Department of Chemistry, Faculty of Mathematics and Natural Sciences, Universitas Padjadjaran, Indonesia. ${ }^{3}$ Research Center of Molecular Biotechnology and Bioinformatics, Universitas Padjadjaran, Indonesia. ${ }^{4}$ Doctoral Program in Biotechnology, School of Postgraduate Studies, Universitas Padjadjaran, Indonesia. Email: t.subroto@unpad.ac.id
\end{abstract}

Received: 28 October 2016, Revised and Accepted: 24 January 2017

ABSTRACT

Objective: Despite the availability of the commercial rapid tests of chikungunya, the difference of pathogen's genotypes amongst different countries has created some causes for concern. It is found that the sensitivity of the current chikungunya rapid tests on Asian strain was only $20.5 \%$, as compared to 90.3\% when tested on the African phylogroup. Therefore, the development of diagnostics that is specific for the current strain circulating in the country is important to be done. The cryo-electron microscopy (cryo-EM) structures of antigen-antibody complex can be used as an insightful structural basis to the development of the tailored antibody for diagnostics purposes. However, cryo-EM structures usually were resolved in low resolution, thus some sterical clashes between residues are expected. This work aims to refine the cryo-EM structures of E1-E2 of chikungunya virus in complex with antibody using molecular mechanics method, to calculate the binding energy of antigen-antibody complex, and to compare it with the experimental results.

Methods: The cryo-EM structures were refined in vacuo by short minimization scheme using AMBER 14. The binding energies were calculated using FireDock and Molecular Mechanics Generalized Boltzmann Surface Area methods.

Results: The results showed that the direct calculation of binding energies of cryo-EM structures reflected high repulsive forces. While the calculation on the refined structure showed lower binding energies. Visual inspections on the complex structures also indicated that the refined structures showed better interactions.

Conclusion: The refinement of cryo-EM structures should be useful to gain more insight into the binding mode of interactions between antigenic protein and antibody, at the atomic level.

Keywords: Antibody, Chikungunya, In silico.

(C) 2017 The Authors. Published by Innovare Academic Sciences Pvt Ltd. This is an open access article under the CC BY license (http://creativecommons. org/licenses/by/4. 0/) DOI: http://dx.doi.org/10.22159/ajpcr.2017.v10s2.19489

\section{INTRODUCTION}

Chikungunya, an arthralgic disease, was first documented in East Africa as an epidemic in 1952-1953 [1,2]. This disease is transmitted to human by Aedes mosquitoes and caused by chikungunya virus (CHIKV) which belongs to Togaviridae family, genus Alphavirus [3]. CHIKV infection has caused a number of outbreaks in East and South Africa and also in Southeast Asia during the past 50 years. Chikungunya has similar symptoms with dengue fever and is characterized by painful and acute syndrome with fever, headache, skin rash, and joint pain (arthralgia) [4]. The latter symptom distinguishes CHIKV from dengue virus [5].

The most recent epidemic of CHIKV infections was recorded in the year of 2000 in Kinshasa (50,000 estimated cases) and 2001-2003 in Indonesia. It has also spread to the Indian Ocean Islands of Mayotte, Mauritius, Réunion, and the Seychelles with 270,000 cases in 20052006 in La Réunion Island), and India with 1.4-6.5 million estimated cases in 2006-2007 [3]. Chikungunya was recently reported to be endemic in Bandung, Indonesia [6].

Although the commercial rapid tests of chikungunya have been available, the difference of pathogen's genotypes among different countries has created some causes for concern. The sensitivity of the current chikungunya rapid tests on Asian strain was only $20.5 \%$ of that of African strain [7]. Therefore, the development of diagnostics that is specific for the current strain circulating in the country is important to be done. The cryo-electron microscopy (cryo-EM) structures of antigen-antibody complex can be used as an insightful structural basis to the development of the tailored antibody for diagnostics purposes. However, cryo-EM structures usually were resolved in low resolution, thus, some sterical clashes between residues are expected. The refinement of cryo-EM structures should be useful to gain better understanding of the intermolecular interactions, at the atomic level.

In 2013, four Fab fragments of monoclonal antibodies, namely, CHIK 9, CHIK-152, $\mathrm{m} 10$, and $\mathrm{m} 242$, were complexed with pseudo-atomic CHIK virus-like particles (VLPs). These antibodies bound to the E1E2 antigenic protein of CHIKV. The cryo-EM structures of the four Fabs have been deposited in Protein Data Bank (PDB) [8]. Different neutralization mechanism between the Fabs was observed. Based on the analysis of these cryo-EM structures, differences on the Fab's footprint Fabs on VLP were shown. The footprints of CHK-9 and m242 Fabs were dominantly localized on the domain A while m10's on the domain B. CHK-152's footprint was spanned at both domains A and B. It was noted that CHK-152 prevents the viral fusion. Three other Fabs neutralized CHIKV infectivity by blocking the cellular binding site on the A domain of E2 protein [8]. Nevertheless, a detailed picture of the atomic interactions between antigen and antibody in these cryo-EM structures are remain unclear.

The objectives of this study were to refine the cryo-EM structures of E1-E2 of CHIKV in complex with antibody using molecular mechanics method, to calculate the binding energy of antigen-antibody complex, and to compare it with the experimental results. 


\section{METHODS}

\section{Cryo-EM structures}

Cryo-EM structures of antigen-antibody complex of chikungunya which used in this study were downloaded from PDB. The PDB IDs for CHK-9, CHK-152, m10, m242, and E1-E2 CHIKV are 3J2Y, 3J30, 3J2Z, 3J2X, and $3 \mathrm{~J} 2 \mathrm{~W}$, respectively [8].

\section{Molecular mechanics refinement of Cryo-EM structures}

The cryo-EM structures of antigen-antibody complex of E1-E2 CHIKV were minimized in vacuo by 250 steps of steepest descent and followed by 250 steps conjugate gradient methods, using sander module in AMBER 14 [9]. The cutoff for non-bonded distance was $12 \AA$.

\section{Binding energy calculations}

Two methods were used to calculate the binding energy of antigenantibody, i.e., FireDock [10] and Molecular Mechanics Generalized Boltzmann Surface Area (MM/GBSA) method implemented in MMPBSA.py [11]. In FireDock, options of antibody-antigen complex type, 100 cycles, and full refinement level were selected. In MM/GBSA calculation, salt concentration of $150 \mathrm{mM}$ was used.

\section{RESULTS}

First, the antigen-antibody complex structures were prepared by combining the coordinates of E1-E2 CHIKV (PDB ID 3J2W) with that of the antibody. Then, the complex structures were submitted to FireDock server (http://bioinfo3d.cs.tau.ac.il/FireDock/index.html) to get the calculated binding energy. The result showed that the repulsive van der Waals force dominated the binding energy of $\mathrm{m} 10$ (Table 1). Hydrogen bond energies, one of the most important non-covalent bonds, were also generally weak in all of the complexes. This indicated that the atomic conformations in cryo-EM structures were not in a favorable position.

Furthermore, all of the antigen-antibody complexes were refined by molecular mechanics method. A short minimization (500 steps) was conducted in vacuo, to remove any bad contacts or sterical clashes that may lead to the repulsive forces. It is showed that when the refined complexes were submitted to FireDock server, the calculated binding energies improved (Table 2). It is worth noting that a high repulsive force in $\mathrm{m} 10$ system was removed. In general, the global binding energy, attractive van der Waals force, and hydrogen bond energy of all the refined systems were stronger than that of the initial conformations of cryo-EM structures.

The changes of energies reflected that the atomic conformations after minimization were in more favorable positions than before. Visual inspection on the refined structure showed no sterical clashes among the side chains of antigen and antibody. Fig. 1 shows that the primary amino group of Lys733 was in a very near distance with the side chain of Asp600 (0.938 $\AA$ ). Furthermore, it is noted that the distance has increased to $3.895 \AA$ after being refined by geometry minimization using molecular mechanics method.

However, despite the stronger binding energy (Table 2), the correlation between calculated binding energy and experimental effective concentration 50 (EC50) was not satisfying $\left(\mathrm{R}^{2}=0.1224\right)$. A possible reason behind this result was due to the simplified solvation model that is used in FireDock, i.e., atomic contact energy approach [10]. Therefore, the binding energy of the refined complexes were further calculated by MM/GBSA method, which uses a continuum solvent model (Table 3).

While FireDock shows $\mathrm{m} 10$ as the most active Fab, MM/GBSA was successfully identified CHK-152 as the best Fab with the strongest binding to E1-E2 CHIKV, although the third and fourth ranks of Fab were not really in agreement with the experimental values. However, the correlation coefficient of MM/GBSA was better than FireDock in this case.

\section{DISCUSSION}

Based on the binding energy, CHIK-152 was identified as the best antibody bound to E1-E2 CHIKV. This is in agreement with the fact that CHIK-152 has a better neutralization effect than $\mathrm{m} 10$ [8]. Pal and colleagues also reported that CHIK-152 prevented CHIKV infection up to $90 \%$ in all phylogroups, i.e., African and Asian strains [12]. The binding profile of CHIK-152 on E1-E2 CHIKV, at the atomic level, should be useful to provide structural basis in designing a specific antibody that can be used for a

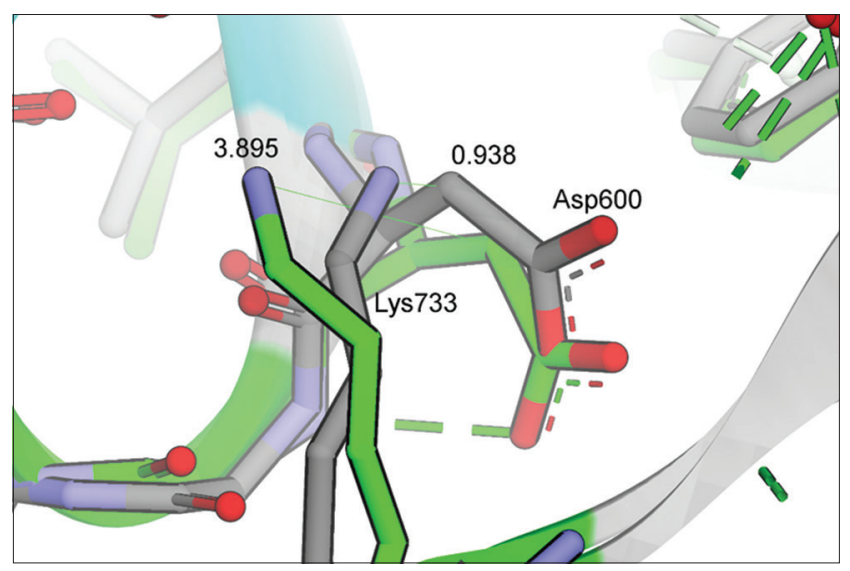

Fig. 1: Superimposition of the refined cryo-electron microscopy structure (green-colored carbon stick) on the initial structure (gray-colored carbon stick)

Table 1: Direct calculation of binding energy of cryo-EM structures using FireDock [8]

\begin{tabular}{lllllll}
\hline Fab & PDB ID & Experimental EC50 $(\boldsymbol{\mu g} / \mathbf{m l}) *$ & $\begin{array}{l}\text { Binding } \\
\text { energy (kcal/mol) }\end{array}$ & $\begin{array}{l}\text { Attractive } \\
\text { VdW (kcal/mol) }\end{array}$ & $\begin{array}{l}\text { Repulsive } \\
\text { VdW (kcal/mol) }\end{array}$ & $\begin{array}{l}\text { H-bond energy } \\
\text { (kcal/mol) }\end{array}$ \\
\hline CHIK-152 & 3J30 & 0.013 & -9.7 & -13.94 & 11.70 & -0.81 \\
m10 & 3J2Z & 2.9 & 189.46 & -10.75 & 335.50 & -2.26 \\
CHIK-9 & 3J2Y & 18 & 22.85 & -25.27 & 11.85 & -2.07 \\
m242 & 3J2X & 20 & -6.98 & -7.40 & 0.75 & -1.11 \\
\hline
\end{tabular}

EC: Effective concentration, cryo-EM: Cryo-electron microscopy, CHIK: Chikungunya virus, PDB: Protein Data Bank

Table 2: Calculation of binding energy of the refined cryo-EM structures using FireDock [8]

\begin{tabular}{lllllll}
\hline Fab & PDB ID & Experimental EC50 $(\boldsymbol{\mu g} / \mathbf{m l}) *$ & $\begin{array}{l}\text { Binding } \\
\text { energy (kcal/mol) }\end{array}$ & $\begin{array}{l}\text { Attractive } \\
\text { VdW (kcal/mol) }\end{array}$ & $\begin{array}{l}\text { Repulsive } \\
\text { VdW (kcal/mol) }\end{array}$ & $\begin{array}{l}\text { H-bond energy } \\
\text { (kcal/mol) }\end{array}$ \\
\hline CHIK-152 & 3J30 & 0.013 & -76.54 & -55.89 & 17.19 & -9.57 \\
m10 & 3J2Z & 2.9 & -100.64 & -74.59 & 18.44 & -12.35 \\
CHIK-9 & 3J2Y & 18 & -56.19 & -69.94 & 17.32 & -7.03 \\
m242 & 3J2X & 20 & -89.78 & -57.95 & 16.98 & -6.69 \\
\hline
\end{tabular}

EC: Effective concentration, cryo-EM: Cryo-electron microscopy, CHIK: Chikungunya virus, PDB: Protein Data Bank 
Table 3: Binding energies calculated by FireDock and MM/GBSA as compared to the experimental EC50 [8]

\begin{tabular}{|c|c|c|c|c|c|}
\hline Fab & Experimental EC50 $(\mu \mathrm{g} / \mathrm{ml})^{*}$ & $\begin{array}{l}\text { FireDock } \\
\text { binding energy (kcal/mol) }\end{array}$ & FireDock $\mathbf{R}^{2}$ & $\begin{array}{l}\text { MM/GBSA } \\
\text { binding energy (kcal/mol) }\end{array}$ & $\begin{array}{l}\text { MM/GBSA } \\
\mathbf{R}^{2}\end{array}$ \\
\hline CHIK-152 & 0.013 & -76.54 & 0.1224 & -3.45 & 0.7306 \\
\hline $\mathrm{m} 10$ & 2.9 & -100.64 & & 17.39 & \\
\hline CHIK-9 & 18 & -56.19 & & 35.26 & \\
\hline $\mathrm{m} 242$ & 20 & -89.78 & & 25.62 & \\
\hline
\end{tabular}

EC: Effective concentration, cryo-EM: Cryo-electron microscopy, CHIK: Chikungunya virus, PDB: Protein Data Bank, MM/GBSA: Molecular Mechanics Generalized Boltzmann Surface Area

sensitive diagnostic agent, or even therapeutics. A tailored antibody can be further developed into a paper-based diagnostics or biosensors [13]. Recently, four new methods to immobilize antibody on the nitrocellulose were introduced to support the rapid test developer [14].

In this study, binding energy calculated by MM/GBSA has better correlation coefficient with the experimental values, as compared to FireDock. This result is in agreement with the previous studies which showed that MM/GBSA method is often useful to refine the binding scores resulted from docking-based virtual screening [15]. Nevertheless, a better conformational sampling for MM/GBSA method should be able to improve the accuracy of binding energy calculation. This can be achieved by molecular dynamics simulations.

\section{CONCLUSION}

This study showed that the cryo-EM structures should be refined first before any structural analysis, especially for the purpose of designing antibody. Sterical clashes in the initial cryo-EM structure were observed by visual inspection. Structural refinement by short minimization using molecular mechanics method was able to improve the binding energy between antigen and antibody. However, the correlation between the calculated binding energy resulted from FireDock, and the experimental EC50 was not satisfying. Recalculation of binding energy using MM/GBSA has improved the correlation coefficient between the calculated and experimental values from 0.1224 to 0.7306 . This study is an important initiative to the further development of antibody for a diagnostic agent.

\section{ACKNOWLEDGMENT}

We would like to thank Computational Chemistry and Bioinformatics (CCB) Laboratory, Universitas Padjadjaran, Jalan Singaperbangsa No. 2 Bandung, to provide computing facilities for this work.

\section{REFERENCES}

1. Charrel RN, de Lamballerie X, Raoult D. Chikungunya outbreaks - The globalization of vectorborne diseases. NEngl J Med 2007;356(8):769-71.
2. Schwartz O, Albert ML. Biology and pathogenesis of chikungunya virus. Nat Rev Microbiol 2010;8(7):491-500.

3. Sourisseau M, Schilte C, Casartelli N, Trouillet C, Guivel-Benhassine F, Rudnicka $\mathrm{D}$, et al. Characterization of reemerging chikungunya virus. PLoS Pathog 2007;6:804-17.

4. Burt FJ, Rolph MS, Rulli NE, Mahalingam S, Heise MT. Chikungunya: A re-emerging virus. Lancet 2012;379(9816):662-71.

5. Pialoux G, Gaüzère BA, Jauréguiberry S, Strobel M. Chikungunya, an epidemic arbovirosis. Lancet Infect Dis 2007;7(5):319-27.

6. Kosasih H, De Mast Q, Widjaja S, Sudjana P, Antonjaya U, Ma'roef C, et al. Evidence for endemic chikungunya virus infections in Bandung, Indonesia. PLoS Negl Trop Dis 2013;7(10):e2483.

7. Kosasih H, Widjaja S, Surya E, Hadiwijaya SH, Butarbutar DP, Jaya UA, et al. Evaluation of two IgM rapid immunochromatographic tests during circulation of Asian lineage chikungunya virus. Southeast Asian J Trop Med Public Health 2012;43(1):55-61

8. Sun S, Xiang Y, Akahata W, Holdaway H, Pal P, Zhang X, et al. Structural analyses at pseudo atomic resolution of chikungunya virus and antibodies show mechanisms of neutralization. Elife 2013;2:e00435.

9. Case DA, Babin V, Berryman JT, Betz RM, Cai Q, Cerutti DS. AMBER 14. San Francisco: University Of California; 2014

10. Andrusier N, Nussinov R, Wolfson HJ. Firedock: Fast interaction refinement in molecular docking. Proteins 2007;69(1):139-59.

11. Miller BR $3^{\text {rd }}$, Mcgee TD Jr, Swails JM, Homeyer N, Gohlke H, Roitberg AE. MMPBSA.py: An efficient program for end-state free energy calculations. J Chem Theory Comput 2012;8(9):3314-21.

12. Pal P, Dowd KA, Brien JD, Edeling MA, Gorlatov S, Johnson S, et al. Development of a highly protective combination monoclonal antibody therapy against chikungunya virus. PLoS Pathog 2013;9(4):e1003312.

13. Liu Y, Liu Y, Mernaugh RL, Zeng X. Single chain fragment variable recombinant antibody functionalized gold nanoparticles for a highly sensitive colorimetric immunoassay. Biosens Bioelectron 2009;24(9):2853-7.

14. Holstein CA, Chevalier A, Bennett S, Anderson CE, Keniston K, Olsen $\mathrm{C}$, et al. Immobilizing affinity proteins to nitrocellulose: A toolbox for paper-based assay developers. Anal Bioanal Chem 2016;408(5):1335-46.

15. Wang J, Hou T, Xu X. Recent advances in free energy calculations with a combination of molecular mechanics and continuum models. Curr Comput Aided Drug Des 2006;2:95-103. 